TERAHERTZ

\title{
wireless communications
}

\section{III/V photonics: a basic THz communications technology compatible with fibre-optic networks}

\author{
Guillaume DUCOURNAU, IEMN, Université de Lille - guillaume.ducournau@iemn.univ-lille1.fr
}

The growing capacity of wireless networks, driven mainly by new uses and growing mobility, has created a need for new communication systems. The terahertz $(\mathrm{THz})$ frequency range, corresponding to long optical wavelengths (very far infrared) and very high-frequency electronics (at the limit of a transistor's intrinsic capacity), is a prime candidate for these applications.

$\mathrm{I}$ he need to communicate has always spurred the development of technology for transmitting information. Copper wiring, for instance, with its limited transmission capacity, has been outstripped by technologies that send light beams down thin optical fibres over long distances (undersea or metropolitan networks), while on a smaller scale, optical links are replacing data buses in high-speed calculators. These technologies are increasingly reliant on so-called silicon photonics, as its high level of integration brings far greater compactness.

Internet Protocol (IP) traffic is constantly increasing, with monthly traffic expected to reach 130 exabytes
( $1 \mathrm{~EB}=10^{18}$ bytes) by 2018 [1], and the next revolution for the first half of the 21st century will therefore be the advent of technologies allowing for the massive development of wireless communication. However, although changes in use mean that the greatest growth is expected to be in wireless communication networks, these are still the weakest link in terms of data rate, even though more and more online services are now mainstream.

Since G. Marconi's experiments in the early days of radio, carrier frequencies (carrier waves convey information) have become ever higher, and wireless transmission systems will be exploiting the terahertz range of frequencies $\left(1 \mathrm{THz}=10^{12}\right.$ hertz) by 2020 [2],

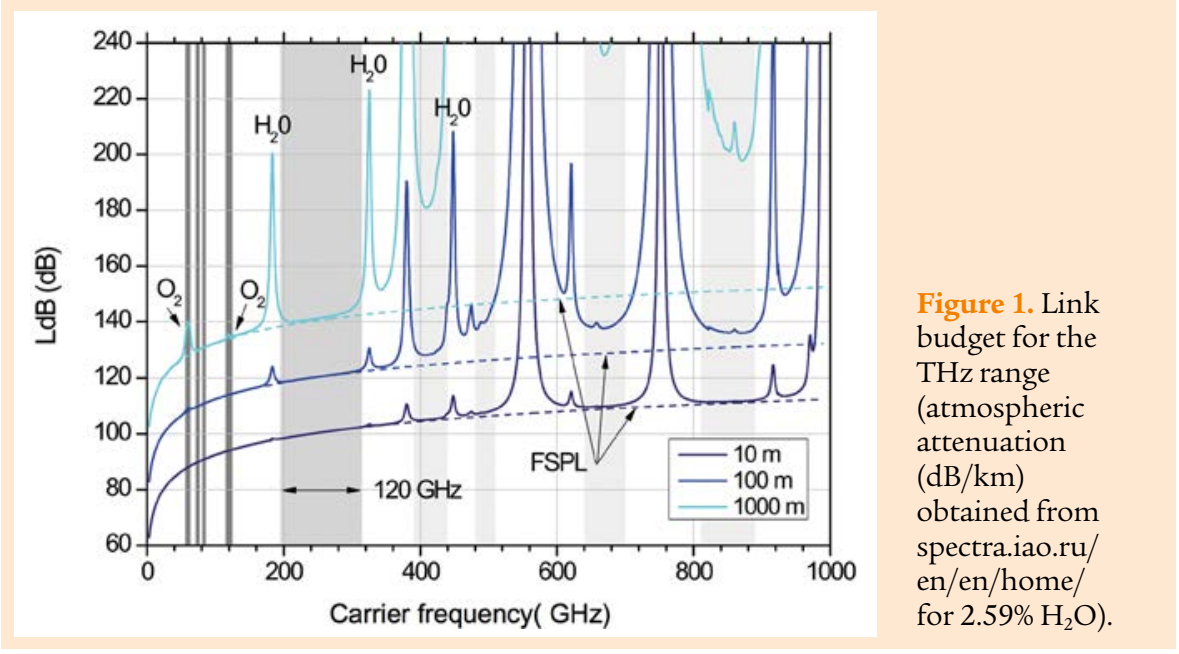

if only to increase communication network capacity between each base station. The Shannon-Hartley theorem, which allows us to calculate channel capacity $\left(C=B \log _{2}(1+S / N)\right.$, where $C$ is the capacity in bits/s, $B$ the bandwidth in $\mathrm{Hz}$, and $S / N$ the signalto-noise ratio), reminds us that any increase in capacity must be matched by an increase in bandwidth $B$. The problem is that the electromagnetic spectrum is becoming increasingly crowded. This led to the recent opening of new frequency bands, first at $60 \mathrm{GHz}$, then in the $E$ band in the $71-76 \mathrm{GHz}$ and $81-86 \mathrm{GHz}$ ranges $\left(1 \mathrm{GHz}=10^{9}\right.$ hertz $)$. However, these new frequency bands only offer data transfer rates of around 7 Gbps per subband (although this could increase to around $10 \mathrm{Gbps}$ with advanced signal coding), and operating frequencies will have to increase beyond 100 $\mathrm{GHz}$ if we are to achieve rates in the order of several dozen Gbps. Circuits generally have a relative bandwidth of just 10 or $15 \%$ with respect to the central frequency. As a result, the $\mathrm{THz}$ frequency range $(0.3-30 \mathrm{THz}$ for optical physicists, and 0.1-10 THz for electronics engineers), has been the focus of attention for several years now, as researchers attempt to resolve the problem of ultra-fast wireless communication. Moreover, with the development 


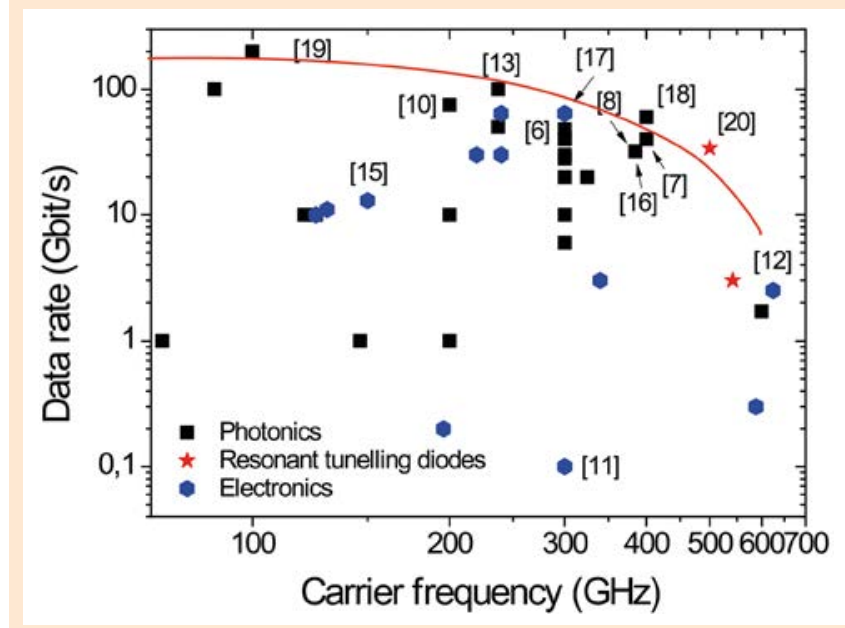

Figure 2. State of the art (data rate/ carrier frequency) in $\mathrm{THz}$ communication. of fibre-to-the-home (FTTH) passive optical networks (PONs), the convergence of radio and fibre technologies is even more relevant today, in the form of fibre to the antenna (FTTA).

Once the technological building bricks have been identified, we will still need to identify the ad hoc frequencies for free-space propagation. Atmospheric absorption is restricted to isotropic path loss (link budget for omnidirectional antennae) up to a distance of $1 \mathrm{~km}$ (Fig. 1) for frequencies at the upper limit of the millimetric band, above $220 \mathrm{GHz}$. The major advantage here is that bands above 275 $\mathrm{GHz}$ have not yet been allocated to specific applications. Standardization attempts are currently underway at the Institute of Electrical and Electronics
Engineers (IEEE), where an interest group is looking into the introduction of a standard for $100 \mathrm{Gbps}$ at these frequencies [3].

We can therefore confidently predict that, by 2020 , the $\mathrm{THz}$ spectrum will have been explored with a view to using it for ultrafast wireless communications. We can reasonably assume that the data rate for commercial wireless communications will reach 100 Gbps within the next decade. This level will be vital for the realtime transmission of high-definition video flows. For example, the output signal of an HD camera has a rate of 1.5 Gbps, so compression techniques are required to adapt it to the limited bandwidth of conventional transmission channels (approx. $20 \mathrm{Mbps}$ ).

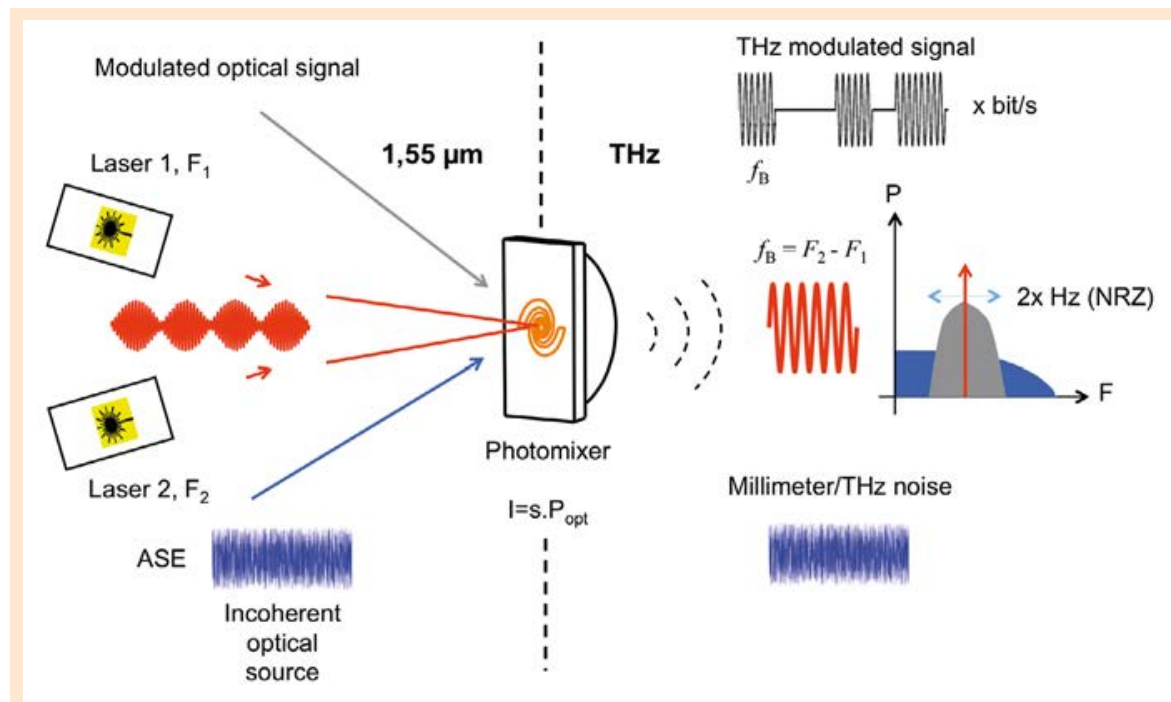

Figure 3. Photomixing technique for generating a THz signal in continuous mode (red), as either a non-return-to-zero (NRZ) amplitude-modulated signal (grey), or an incoherent signal (blue).

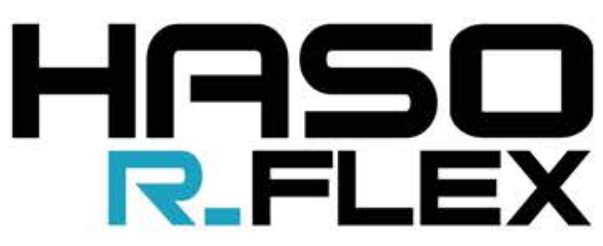

The most practical and cost effective metrology tool for characterizing optical components such as large concave

mirrors, lenses, complex optical systems, and beam expanders
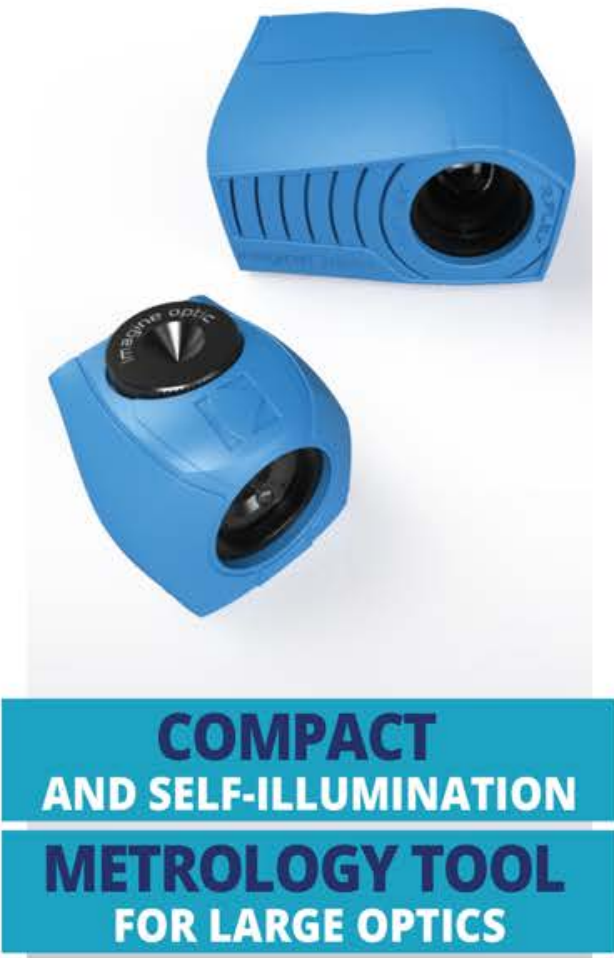

EASY TO USE AND VERSATILE

LARGE DYNAMIC AND HIGH ACCURACY

Contact us for more details: contact@imagine-optic.com or +33164861560 


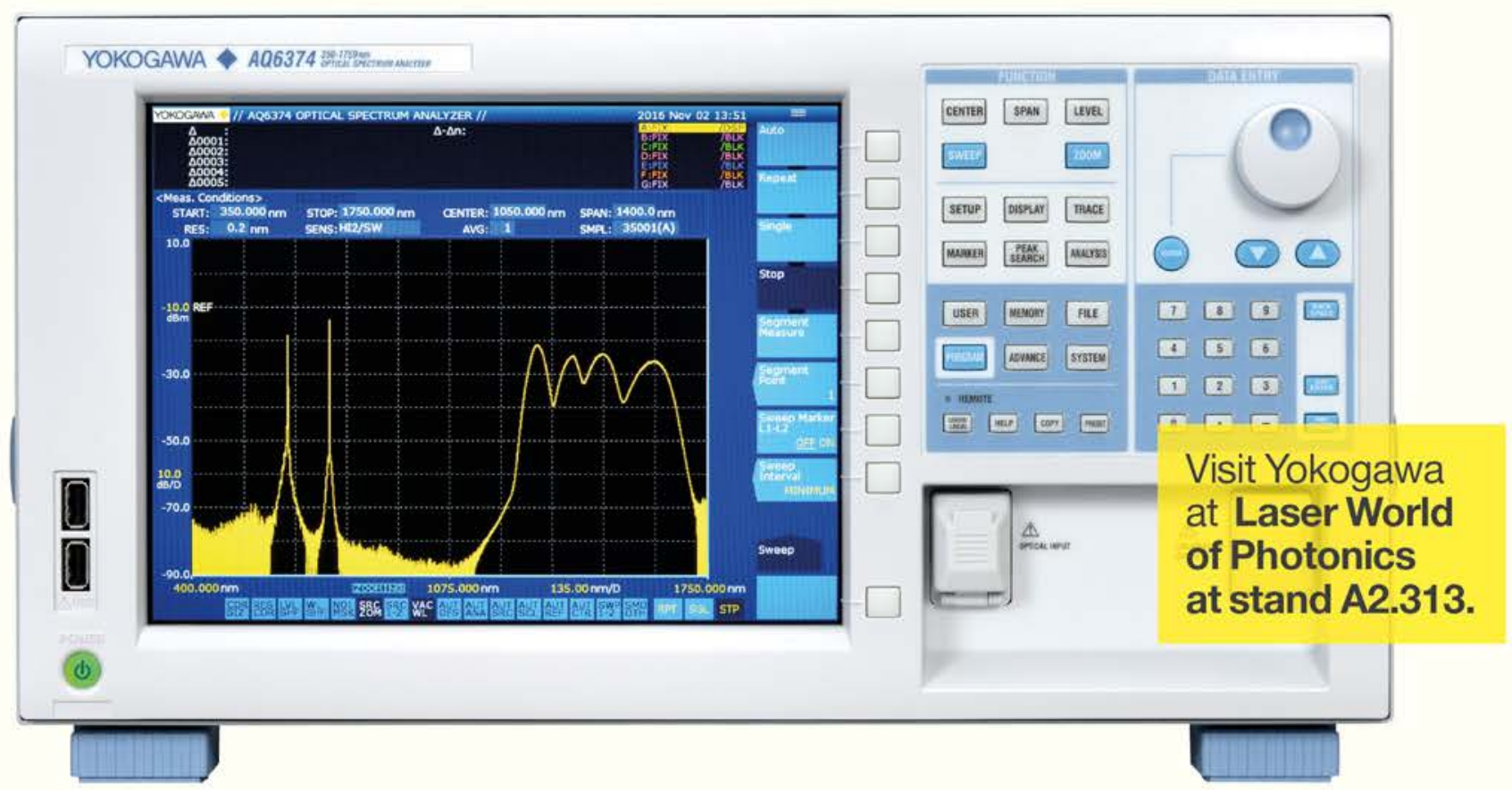

\section{An OSA for every application}

\section{Trusted instruments for world class optical performance}

There is an AQ6370 series Optical Spectrum Analyser (OSA) which satisfies your specific measurement needs in the range from visible light to mid-infrared ( 350 to $3400 \mathrm{~nm}$ ).

- The highest wavelength resolution up to $10 \mathrm{pm}$

- The widest measurement power range up to $110 \mathrm{~dB}$

- The highest sensitivity down to $-90 \mathrm{dBm}$

- An unmatched close-in dynamic range: up to $78 \mathrm{~dB}$

- The free space optical input

For your specific application,

contact us now and get a demo in your lab. 
$4 \mathrm{~K}$ televisions, which have four times as many pixels as the usual HD models, require realtime rates above 6 Gbps. Beyond broadcast-type applications or communication between base stations in the future $6 \mathrm{G}$ networks, wireless video transfer could be used for video transmission, or to rid operating theatres of the clutter of cables. The development of THz-range wireless communications will therefore require the development of terminal equipment (sources, amplifiers, detectors, antennae) that afford a power margin compatible with the link budget.

A range of different technologies have been or are in the process of being developed in laboratories to produce the first series of demonstrators, just as we saw with fibre-optic communication during early age in the 70-80's. Although both electronic and photonic solutions have been introduced, it is clear from the current state of the art (Fig. 2) that the main technologies behind these demonstrators have been taken straight from photonics, namely ultrafast InGaAs/InP uni-travelling-carrier photodiodes (UTC-PDs). Originally developed by the Nippon Telegraph and Telephone Corporation (NTT) in Japan for multi-stage optical reception at $40 \mathrm{Gbps}$, these photodiodes have been pushed to their very limits, making it possible to generate signals of up to $2.5 \mathrm{THz}$ [4]. In the frequency bands appropriate for communication applications (around $300 \mathrm{GHz}$ ), levels in the order of $\mathrm{mW}$ have been reached [5]. Combining the tunable feature of optical beams in photonics with the photomixing technique (Fig. 3) makes it possible to transfer the techniques for generating vectorial optical fields developed in 2000-2010, and therefore technologically mature, to the $\mathrm{THz}$ range. Ultrafast photodiodes could therefore directly bridge the gap between fibre optics and high-speed radio networks (convergence of optical and radio technologies; see Fig. 4).

The advantage of techniques borrowed from photomixing is that they produce a very high modulation index of the optical wave, and thus of the $\mathrm{THz}$ wave generated by mixing. Moreover, if extra wavelengths are added, these techniques make it relatively easy to undertake multifrequency communication, which is extremely difficult to transpose to electronics. Coupling optical/THz techniques with $\mathrm{THz}$ receivers using Schottky

\section{Your partner in Laser and Optics developments}

- Laser micromachining with ultra-fast lasers

- Custom \& innovative fiber lasers

- PCF fiber interfacing

- Opto-electronic devices

- Custom optical systems

Meet us at

Laser World of Photonics booth \#B3 - 336

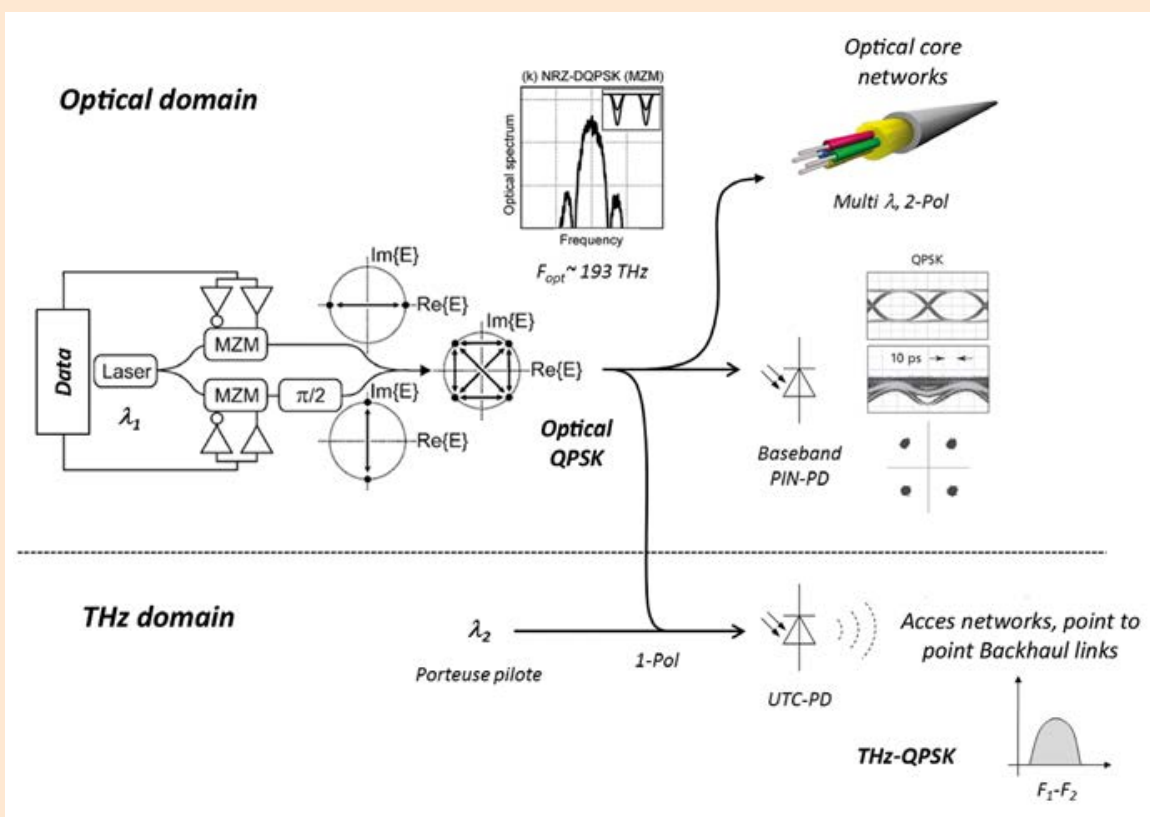

Figure 4. Convergence of optical and radio technologies: a modulated optical wavelength associated with a pilot carrier allows for the transition from optical vectorial modulation (phase coding, like quadrature phase shift keying (QPSK) where 4 phase states are used to encode the optical signal) to QPSK in the THz range. 


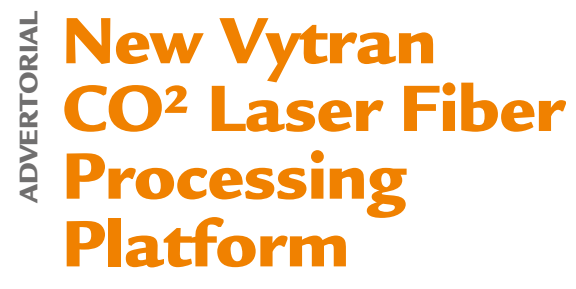

Thorlabs' Vytran ${ }^{\mathrm{TM}}$ Optical Fiber Glass Processors are versatile platforms designed for fabricating fiber splices, tapers, couplers, terminations, and combiners. These systems are ideal for applications involving single mode, multimode, polarization-maintaining, photonic crystal, multicore, and other specialty fibers.

The GPX4000LZ is an integrated fiber processing platform equipped with a $40 \mathrm{~W} \mathrm{CO}^{2}$ laser that offers controlled, precise heating of optical fibers. The uniform, high-temperature heating provided by the laser enables users to process glass fibers up to $\varnothing 2 \mathrm{~mm}$ and splice even larger end caps.

Just as with our other Vytran fiber processors, the GPX4000LZ employs True Core Imaging ${ }^{\mathrm{TM}}$ technology to provide high-resolution images for fiber measurement and alignment. Precise control of process parameters via an integrated control system enables highly automated processing for highvolume manufacturing.

This all-in-one platform has two separate optical heads, optimized for $\mathrm{CO}_{2}$ laser splicing and end capping, respectively. Additionally, the user can easily change out the $\mathrm{CO}_{2}$ laser optical head for a filament furnace heat source, enabling use with existing manufacturing processes.

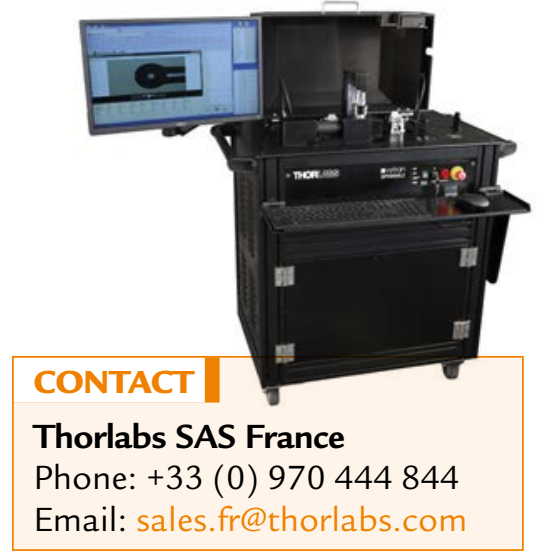

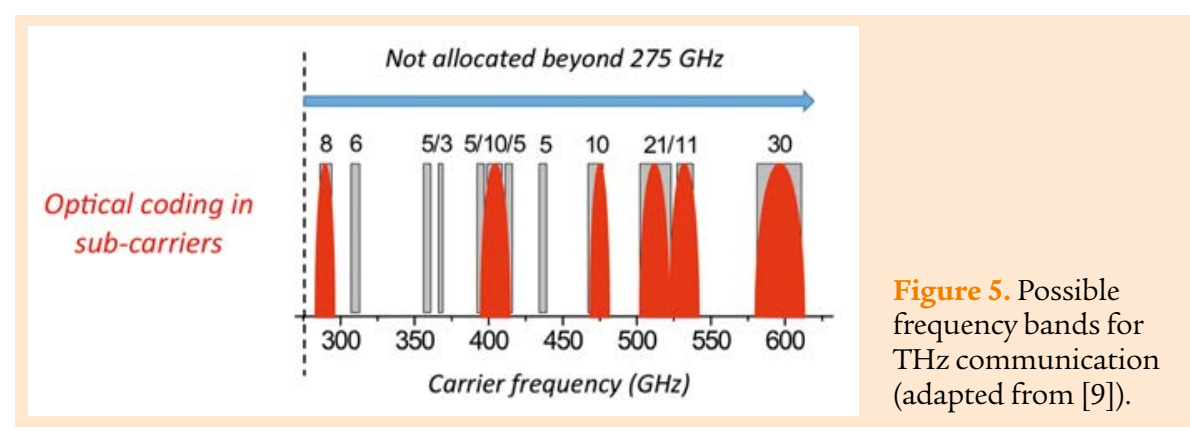

diode mixers (originally developed for radio astronomy) produces data rates for $\mathrm{THz}$ carriers comparable to those of wavelength division multiplexing (WDM) channels over fibre which rely on a multi-color optical signal to increase the total capacity inside the fibre. For example, using technologies developed by NTT, a team in Japan led by $\mathrm{THz}$ communication pioneer Professor Nagatsuma reported realtime transmission rates in amplitude shift keying (i.e. digital amplitude modulation, ASK) amplitude modulation of up to $50 \mathrm{Gbps}$ at $0.3 \mathrm{THz}[6]$ - that is, approximately a thousand times current Wi-Fi! In France, researchers at IEMN have been working on very wide bandwidth communications, and ASK signals of up to $46 \mathrm{Gbps}$ have been measured at $0.4 \mathrm{THz}$ [7] and up to $32 \mathrm{Gbps}$ using complex THz signals (quadrature amplitude modulation) over 25 meters [8]. As optical source phase noise requires corrective signal processing at the receiver end, complex signals are already being used to improve link spectral efficacy in the $\mathrm{THz}$ range, for even though there is a very wide available bandwidth, the presence of observation services (radio astronomy, meteorology) in this frequency range means that future allocations of $\mathrm{THz}$ channels will be in subbands, as illustrated in Figure 5. The use of coherent optical networks and flexgrids coupled with pilot carriers allows multispectral operation to be achieved in the $\mathrm{THz}$ range, as shown in Figure 5. 60-Gbps links operating at $0.2 \mathrm{THz}$ [10] have thus been demonstrated. The race is now on to reach the 100-Gbps data rate, which should become a standard within the coming years for frequency bands above $275 \mathrm{GHz}$.
Technologies derived from electronics are also being developed. The first demonstrators were built using III-V components originally developed for radio astronomy [11,12], as well as high-electron-mobility transistor (HEMT) circuits [13]. It should, however, be noted that the highest rates were first achieved using emission technologies borrowed from photonics, taking advantage of the wide bandwidths associated with opto-electronic devices. As an example, a demonstration was reported at IEMN [8] for up to 32 Gbps transmission over $25 \mathrm{~m}$ at $385 \mathrm{GHz}$ (Fig. 6), using high spectral efficiency. However, even if first links have been achieved 'out-of-the-lab', the limited available output power of photonic devices (currently around $1 \mathrm{~mW}$ at $300 \mathrm{GHz}$ ) will make the future of $\mathrm{THz}$ systems based on photonic transmitters may lie in a combination of solid-state amplifiers (e.g., InP-based heterojunction bipolar transistors (HBTs)) and photomixers. Moreover, the development of $\mathrm{THz}$ links could also benefit from recent advances in silicon photonics. For example, at $180 \mathrm{GHz}$, a Ge-based photomixer on an Si platform can exhibit an equivalent isotropic radiated power (EIRP) of more than $-15 \mathrm{dBm}$ in the $170-190$ $\mathrm{GHz}$ band [14], with the high degree of integration associated with these technologies. As for the likelihood of achieving cheap, low-consumption $\mathrm{THz}$ transmission links in the future, $\mathrm{CMOS}$ technologies have also been considered, and the transmission of several Gbps at $130 \mathrm{GHz}$ has already been achieved for indoor communications [15]. As with early MODEMs and early optical transmission systems, communication in the $\mathrm{THz}$ range holds out the promise of high performance and new uses over the next two decades. 


\section{Acknowledgements}

G. Ducournau would like to thank all the members of IEMN's THz Photonics Group led by Jean-François Lampin, Director of Research at the CNRS. G.D. is also grateful to the French National Research Agency (ANR) for its funding of the "Infra 2013" COM'TONIQ programme on THz communications (ANR - 13 - INFR- 0011-01), and to several other French research programmes and institutions for their support, including Lille University, IEMN (RF MEMS Characterization Centre, Micro-Nano Fabrication Centre, and IEMN-IRCICA Telecom Platform), the CNRS, and the Renatech network. This work was also partly funded by the Equipex FLUX 0017 "Investment for the Future" programme, the Excelsior project, Nord-Pas de Calais Council, the European Regional Development Fund (ERDF), and the "Photonics for Society" state-region project contract (CPER).

\section{FURTHER READING}

[1] The Zettabyte Era: Trends and Analysis, White Paper, CISCO (May 2015)

[2] T.S. Bird, Asia-Pacific Microwave Conference (APMC2011), Keynote, Australia (2011)

[3] http://www.ieee802.org/15/pub/IGthzOLD.html

[4] T. Ishibashi et al., IEEE Journal of Selected Topics in Quantum Electronics 20(6) (2014)

[5] H.-J. Song et al., IEEE Microwave and Wireless Component Letters 22(7) (2012)

[6] T. Nagatsuma et al., IEICE Trans. Electronics E98-C(12), 1060$1070(2015)$

[7] G. Ducournau et al., IEEE Trans. Terahertz Science and Technologies 4(3), 328-337 (2014)

[8] T. Nagatsuma, G. Ducournau, C.C. Renaud, Nature Photonics 10, 371-379 (2016) doi: 10.1038/nphoton.2016.65

[9] S. Priebe et al., IEEE IG THz Group Document, https:// mentor.ieee.org/802.15/dcn/12/15-12-0324-00-0thzinterference-between-thz-communications-and-spaceborne-earth-exploration-services.pdf, accessed 31 January 2016

[10] H. Shams et al., Optics Express 22(19), 23465-23472 (2014)

[11] C. Jastrow et al., Electronics Letters 46(9), 661-663 (2010)

[12] L. Moeller et al., Electronics Letters 47(15), 856-858 (2011)

[13] S. Koenig et al., Nature Photonics 7(12), 977-981 (2013)

[14] S.M. Bowers, et al., Optical Fiber Communications Conference (OFC), USA (2014)

[15] M. Fujishima et al., IEICE Trans. Electronics E98-C(12), 10911104 (2015)

[16] G. Ducournau et al., Electronics Letters 12(11), 915-917 (2015)

[17] I. Kallfass et al., IEICE Trans. Electronics E98-C(12), 1081-1088 (2015).

[18] X. Yu et al., International conference on Photonics in Switching (PS2015), Italy (2015)

[19] X. Li et al., Optics Express 21(16), 187894-187899 (2013)

[20] N. Oshima et al, Electron. Lett. 52(22), 1897 (2016)

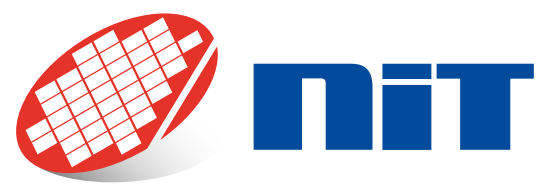

\section{The challenge of Dynamic Range for industrial vision}

In recent years, CMOS imagers have considerably improved their performances in term of resolution, frame rate and sensitivity but the technical problems caused by high contrast or fast changing dynamic scene remain unsolved. New Imaging Technologies proposes its « Native WDR » for both visible and SWIR since its creation thanks to an innovative pixel with logarithmic response. It allows users to cope with saturation and constant set-up changes issues and to provide products that outperform their competitor using classical techniques (multi-exposure, knee point, $\operatorname{lin} / \log , . .$.$) .$

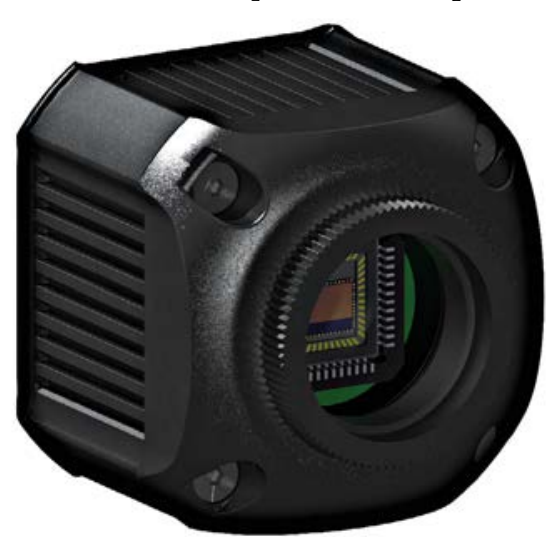
NIT patented technology is indeed the sole to offer local illumination in all point of image (14 bits RAW) and in all images, each obtained in a single exposure. Despite the fact that lighting systems can mitigate the need for dynamic in machine vision, extremely high illumination, high reflecting object, oustide light influence are still a limitation for capturing important details needed for process inspection or monitoring and computer vision. NIT have collaborated with camera or system makers in many high demanding markets such as welding, stereo vision (People counting, 3D measurement), high temperature thermography, process control (laser machining, food, semiconductor...), custom sensor (textile, encoder). Following the trend in camera for more intelligence, NIT has recently launched smart cameras with image processing embedded in FPGA allowing plug and play solution. The smart Widy SWIR 320A and 640A-S and the smart MAGIC $1005 \mathrm{C}$ are offering enhanced vision with $140 \mathrm{~dB}$ and moreover advanced features such AGC, NUC, color management, filters to name a few. These cameras are reconfigurable through serial communication in RS232 or via the camlink. On another front, NIT has also improve its sensor for achieving background suppression, improving vision of textured light or reducing flickering.

\section{CONTACT}

\section{New Imaging Technologies}

Phone: +33(0)164478858 - info@new-imaging-technologies.com

www.new-imaging-technologies.com 